\title{
RADIOCARBON INTERCOMPARISON PROGRAM FOR CHAUVET CAVE
}

\author{
Marie-Thérèse Cuzange ${ }^{1} \bullet$ Emmanuelle Delqué-Količ $^{1} \bullet$ Tomasz Goslar $^{2} \bullet$ \\ Pieter Meiert Grootes ${ }^{3} \bullet$ Tom Higham $^{4} \bullet$ Evelyne Kaltnecker $^{5} \bullet$ Marie-Josée Nadeau $^{3} \bullet$ \\ Christine Oberlin ${ }^{1} \bullet$ Martine Paterne ${ }^{5} \bullet$ Johannes van der Plicht $^{6} \bullet$ Christopher Bronk Ramsey ${ }^{4}$ \\ Hélène Valladas $^{5,7} \cdot$ Jean Clottes $^{8} \bullet$ Jean-Michel Geneste $^{9}$
}

\begin{abstract}
We present the first results of an accelerator mass spectrometry (AMS) radiocarbon intercomparison program on 3 different charcoal samples collected in one of the hearths of the Megaceros gallery of Chauvet Cave (Ardèche, France). This cave, rich in parietal decoration, is important for the study of the appearance and evolution of prehistoric art because certain drawings have been ${ }^{14} \mathrm{C}$ dated to the Aurignacian period at the beginning of the Upper Paleolithic. The new dates indicate an age of about 32,000 BP, which is consistent with this attribution and in agreement with the results from the same sector of the cave measured previously at the Laboratoire des Sciences du Climat et de l'Environnement (LSCE). Six laboratories were involved in the intercomparison. Samples were measured in 4 AMS facilities: Center for Isotope Research, Groningen University, the Netherlands; the Oxford Radiocarbon Accelerator Unit, UK; the Centre de datation par le carbone 14, Univ. Claude Bernard Lyon 1, France (measured by AMS facilities of Poznań University, Poland); and the LSCE, UMR CEACNRS-UVSQ, France (measured by the Leibniz-Labor of Christian-Albrechts-Universität Kiel, Germany).
\end{abstract}

\section{INTRODUCTION}

Chauvet Cave (Ardèche, France), discovered in 1994, is one of the most important sites for the study of the earliest manifestations and development of prehistoric art at the beginning of the Upper Paleolithic.The cave is being studied by an interdisciplinary team set up and directed by $\mathrm{J}$ Clottes (see Clottes et al. 1995; Clottes 2001) from 1998 to the end of 2001 and since then by J-M Geneste (see Geneste 2003). The cave contains more than 420 very well-preserved animal representations including engravings and red and black drawings; some appear isolated and others form parts of large compositions including several figures. The animals represented are naturalistic and very vivid with well-depicted postures. The most abundant animals are rhinoceroses, lions, and mammoths, which are relatively rare in European Paleolithic art. On the other hand, horses and bison are scarcer at Chauvet Cave, while they are more common in Magdalenian art (Züchner 1996). Moreover, evidence for the use of sophisticated techniques (preparation of the wall by scraping, view in perspective, stump drawing, etc.) is very abundant at Chauvet but unusual elsewhere. These characteristics rendered stylistic dating questionable even if some archaeologists proposed the Magdalenian period because of the sophisticated nature of these figures. By chance, the use of charcoal as a pigment for the black drawings allowed the direct dating of some paintings ( 2 fighting rhinoceroses and aurochs of the "Salle Hillaire," a megaloceros of the "Galerie des mégacéros," and a bison from the "Salle du fond"). Several other dates were obtained from torch wipes and charcoal fragments collected in

\footnotetext{
'Centre de datation par le carbone 14, UMR 5138 CNRS, Univ. Claude Bernard Lyon 1, 43 Boulevard du 11 Novembre, 69622 Villeurbanne cedex, France.

${ }^{2}$ Adam Mickiewicz University, Faculty of Physics, Poznań Radiocarbon Laboratory, Ul. Rubież 46, Poznań 61-612, Poland.

${ }^{3}$ Leibniz-Labor, Christian-Albrechts-Universität Kiel, Max-Eyth Str. 11-13, D-24118 Kiel, Germany.

${ }^{4}$ Oxford Radiocarbon Accelerator Unit, Dyson Perrins Building, South Parks Road, Oxford OX13QY, United Kingdom.

${ }^{5}$ LSCE/IPSL, Laboratoire des Sciences du Climat et de l'Environnement, CEA-CNRS-UVSQ, Avenue de la Terrasse, 91198 Gif-sur-Yvette cedex, France.

${ }^{6}$ Center for Isotope Research, Groningen University, Nijenborgh 4, 9747 AG Groningen, the Netherlands. Also: Faculty of Archaeology, Leiden University, PO Box 9515, 2300 RA Leiden, the Netherlands.

${ }^{7}$ Corresponding author. Email: helene.valladas@1sce.cnrs-gif.fr.

${ }^{8} 11$ rue du Fourcat, 09000 Foix, France.

${ }^{9}$ Centre National de Préhistoire, 36 rue du 24ème R.I., 24000 Périgueux, France.
} 
and near ancient hearths. Thus far, about 50 dates obtained by the Laboratoire des Sciences du Climat et de l'Environnement (Gif-sur-Yvette) show that the cave was frequented at least during 2 periods separated by $\sim 5000 \mathrm{yr}$. About $80 \%$ of the dates (including the indicated drawings) correspond to the Aurignacian period, about $32,000 \mathrm{BP}$, and the rest to the Gravettian, some 27,000 BP. These results have an important impact on our knowledge of prehistoric art by demonstrating that the Chauvet cave drawings considerably predated the Magdalenian period, and by showing that sophisticated techniques for wall art were mastered by the Aurignacians at the beginning of the Upper Paleolithic, i.e. far earlier than previously suspected. This means that instead of a steady linear development over millennia, there must have been several periods of intense artistic activity (Clottes et al. 1995, 2001; Valladas et al. 2001, 2005; Geneste 2003).

Besides its great importance for the study of the development of art, Chauvet offers a rare opportunity for obtaining multiple dates, as it contains a great quantity of organic remains (charcoal and bone specimens) - e.g. many charcoal fragments (identified as Pinus sylvestris) are found scattered on the ground near ancient hearths (Figure 1). Some of these specimens were big enough to be divided into fractions to be shared with other laboratories for the purpose of obtaining independent dates. Consequently, at the initiative of the LSCE and the Chauvet team, an interlaboratory prehistoric charcoal dating program was launched as a collaboration between radiocarbon laboratories experienced in dealing with this archaeological material. The importance of this program stems from 2 factors: 1) interlaboratory comparisons involving prehistoric charcoal from sites as old as this one are rare due to the scarcity of such material and 2) new dates obtained by several laboratories should reinforce the already solid database for Chauvet Cave occupation.

The participating laboratories are 1) the Center for Isotope Research, Groningen University, Netherlands; 2) the Oxford Radiocarbon Accelerator Unit, Oxford, UK; 3) the Centre de datation par le carbone 14, Univ. Claude Bernard Lyon 1, France; and 4) the Laboratoire des Sciences du Climat et de l'Environnement, UMR CEA-CNRS-UVSQ, France. The Oxford and Groningen Laboratories did both the chemical treatment and the dating. The Lyon laboratory and the LSCE did their own pretreatment but in the first step of this program submitted the samples to the AMS facilities of Poznań University (Poland) and Leibniz-Labor of Christian-Albrechts-Universität Kiel (Germany), respectively. Measurements of the same samples at the new Artemis AMS facility in France are in progress.

Here, we report the first stage of this intercomparison program, as some laboratories plan to do additional measurements on their own charcoal samples. We intend to extend this program to include other organic remains from Chauvet Cave (such as bone) and to examine the reliability of dates from other prehistoric sites where organic remains are abundant. Moreover, we will now take advantage of the recent developments in the field of sample selection and pretreatment based on chemical and physical investigations (Bronk Ramsey et al. 2004; Cohen-Ofri et al. 2006).

\section{MATERIALS, METHODS, AND RESULTS}

The 3 charcoal fragments labeled GC40, GC41, and GC42 were compact and very well preserved; they were collected from the hearth structure of sector 10.23 (lower floor), located on the ground surface at the left side of the Megaceros gallery, where black drawings abound, and below the representation of a double-maned horse. Each fragment was big enough (3-5 g) to be divided into several fractions weighing between 300 and $500 \mathrm{mg}$, which were sent and dated independently by each laboratory using their own pretreatment methods. Whereas the ABA pretreatment was used by all laboratories, the Oxford team also performed the ABOX treatment (Bird et al. 1999) using 


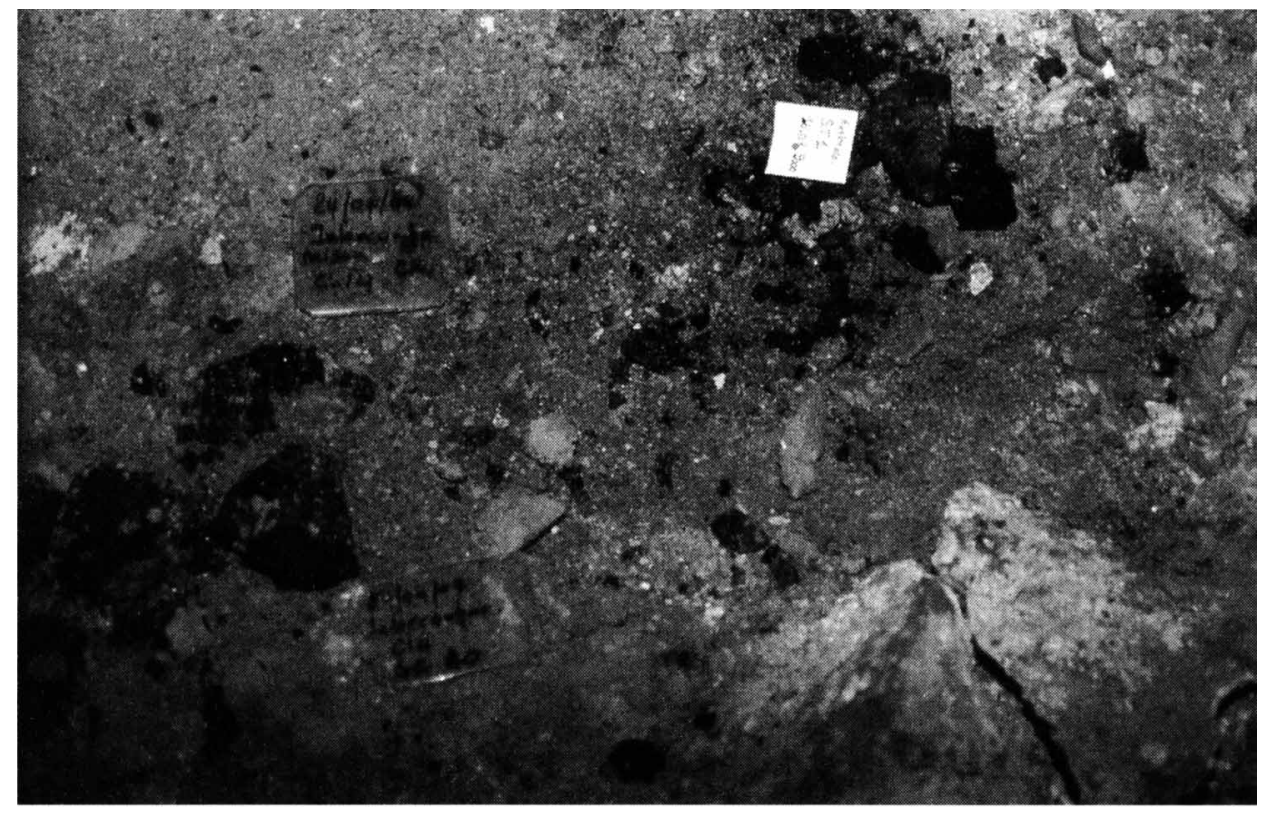

Figure 1 Photograph from the cave floor in the Megaceros gallery showing two of the sampled charcoal pieces

precombustion at $330{ }^{\circ} \mathrm{C}$, or $630{ }^{\circ} \mathrm{C}$ in the case of sample GC40. The alkaline fractions resulting from the pretreatment of GC41 and GC42 were also dated by the Groningen laboratory. Thus far, 29 dates have been obtained: 10 each on GC40 and GC41 and 9 for GC42. The ages and key data for the dating process (such as background values, $\delta^{13} \mathrm{C}$ values, and ${ }^{14} \mathrm{C}$ activities [in pMC] of the samples) are reported in Table 1 . The ${ }^{14} \mathrm{C}$ activities are also plotted in Figure 2.

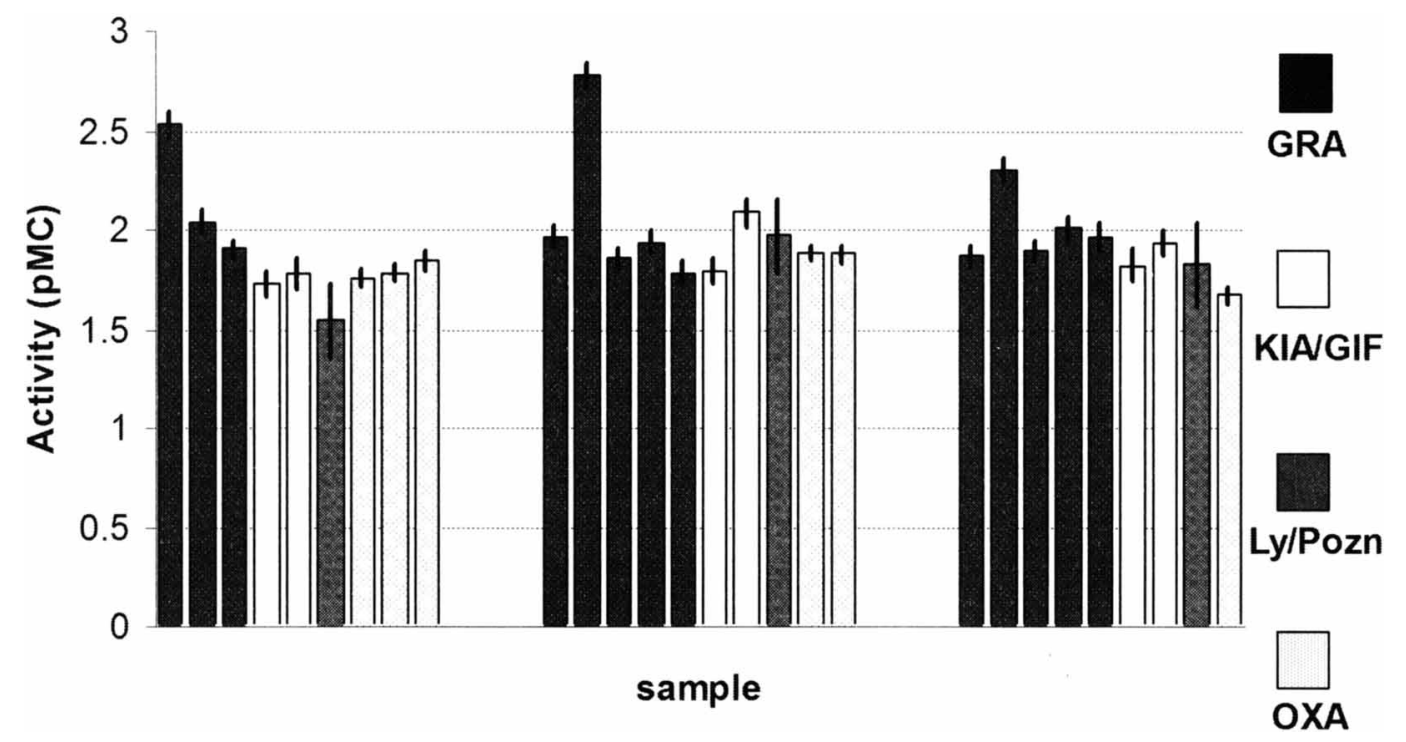

Figure $2{ }^{14} \mathrm{C}$ activities (in pMC) for the measurements of samples GC40, GC41, and GC42 


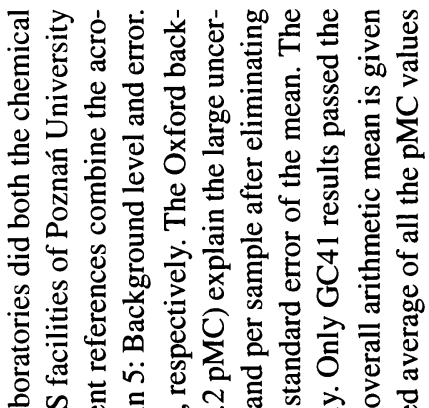

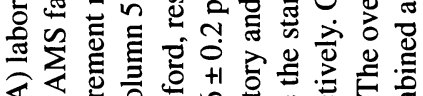

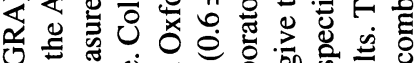

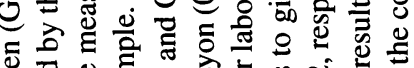

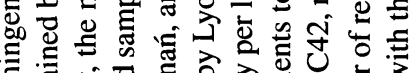

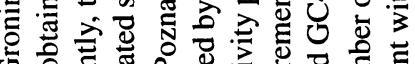
50

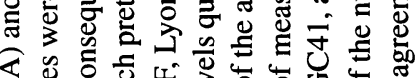

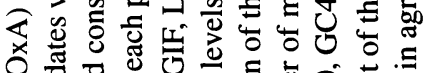

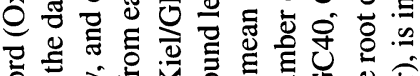

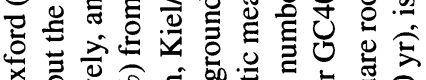

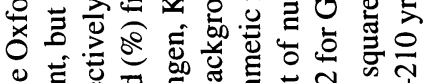

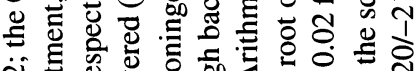

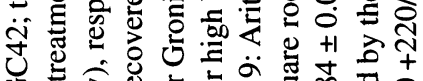

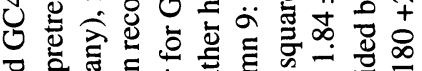

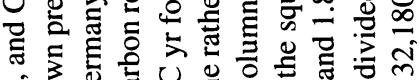

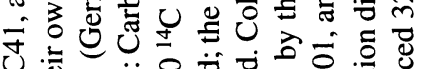

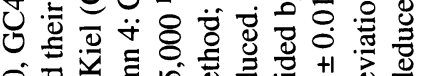

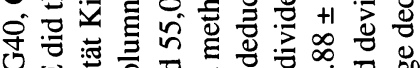

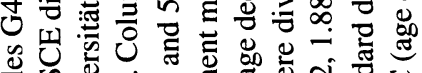

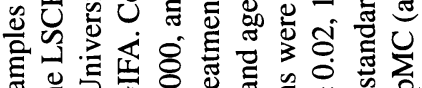
然

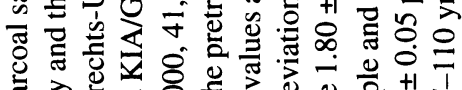

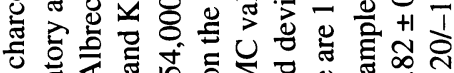

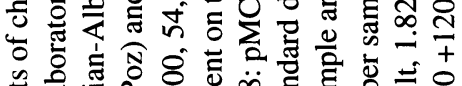

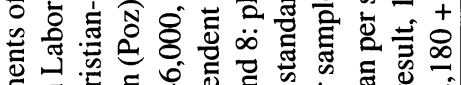

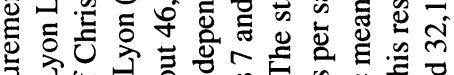

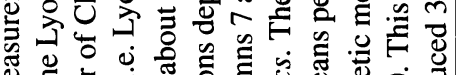

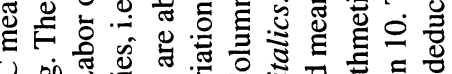

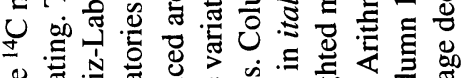

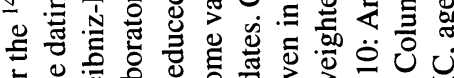

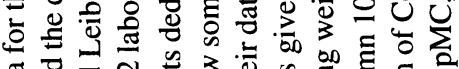

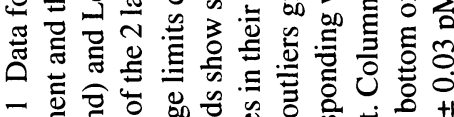

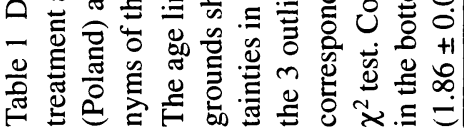

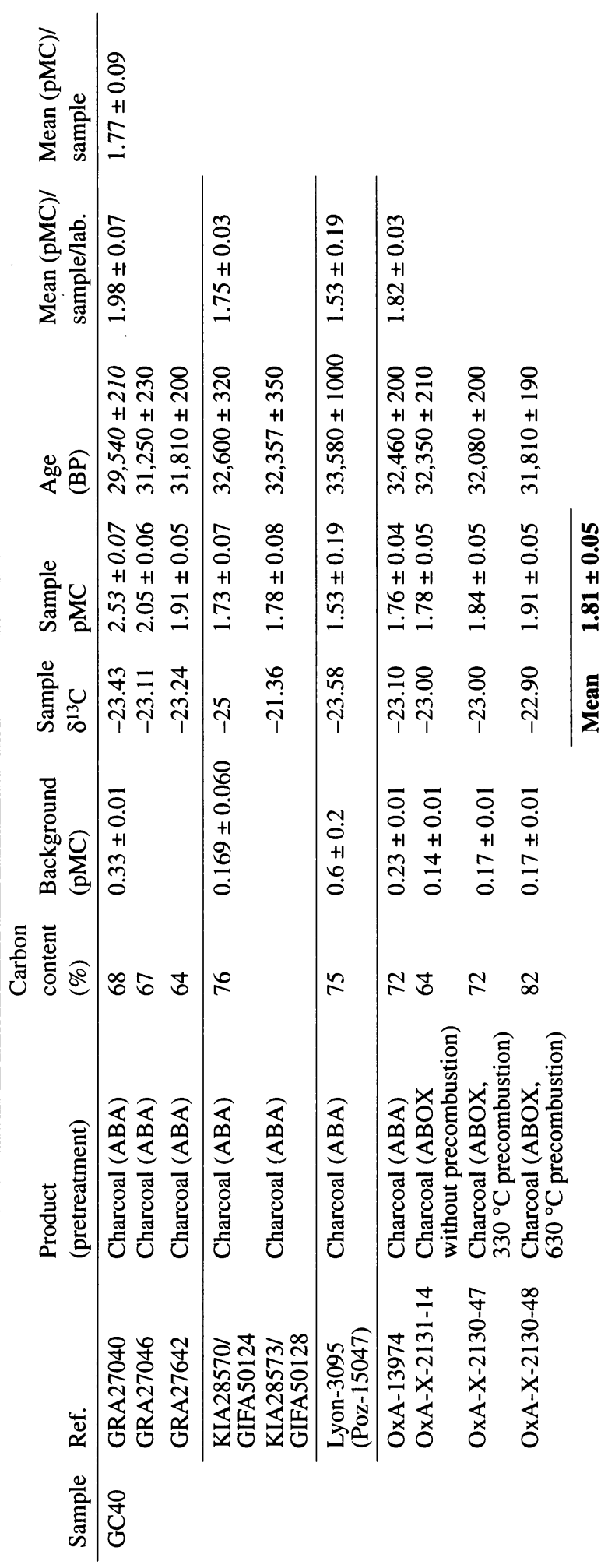




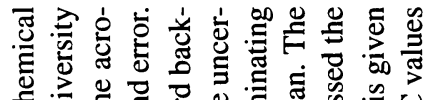

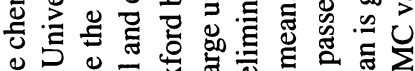
을

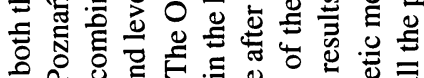

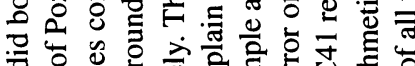
o.

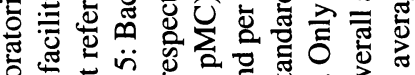

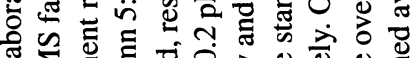

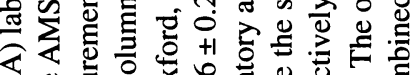
웡을

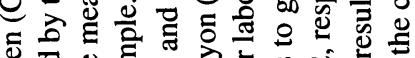

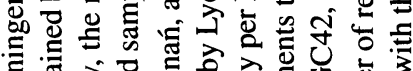

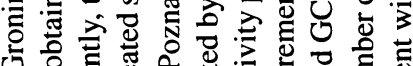

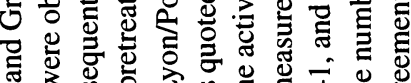
等

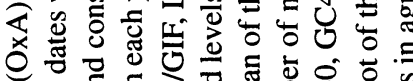

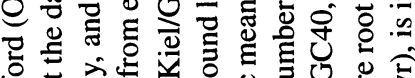

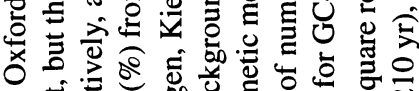
o

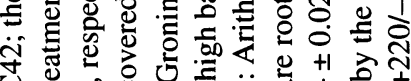

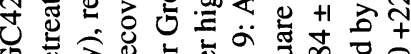

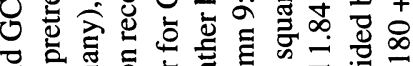
品

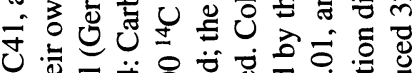

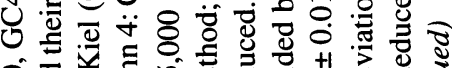

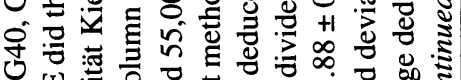

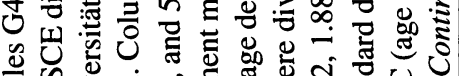

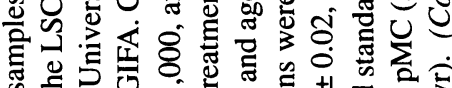

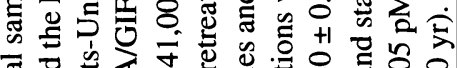

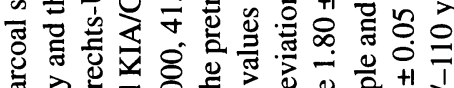

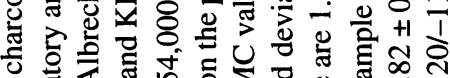

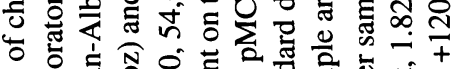

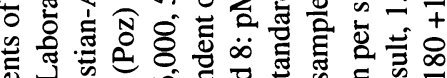

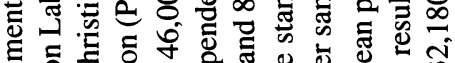

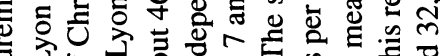

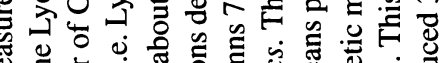

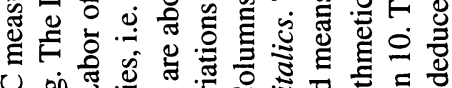

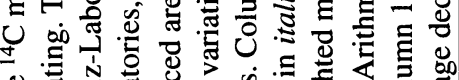

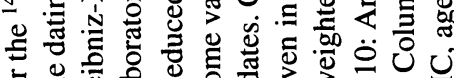

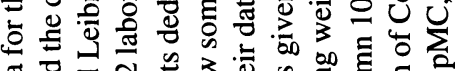

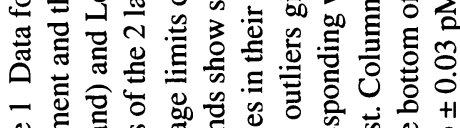

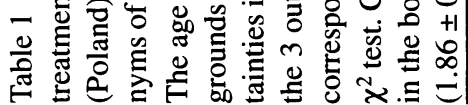

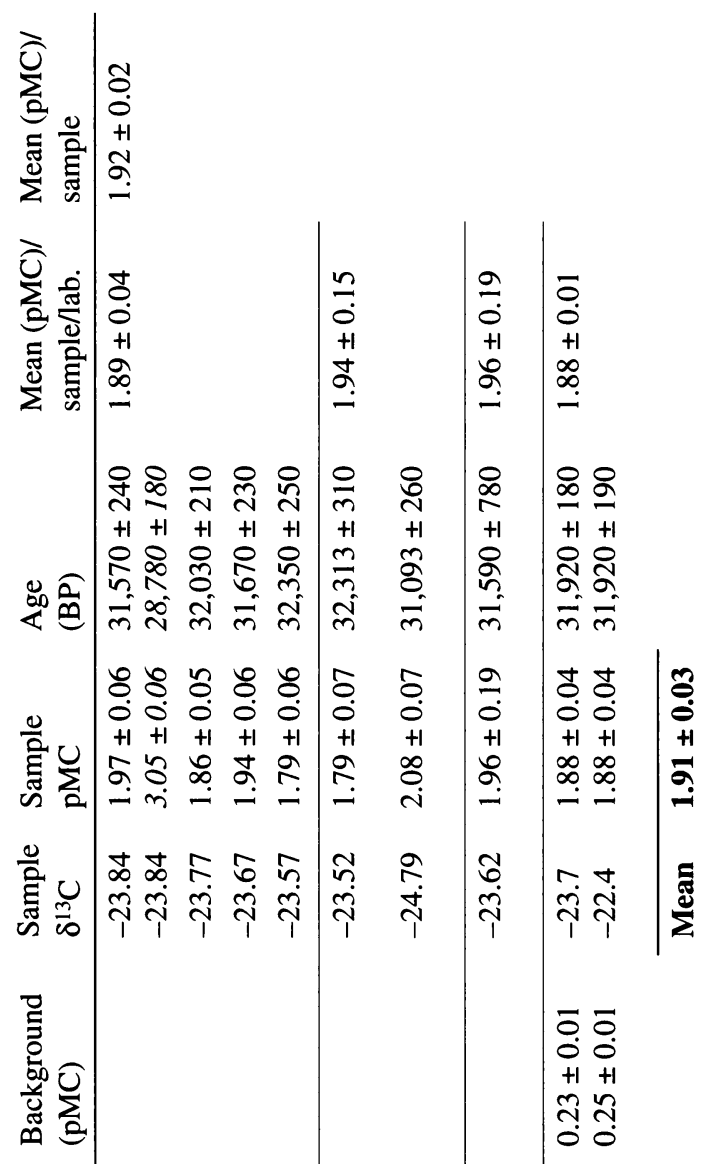

흥
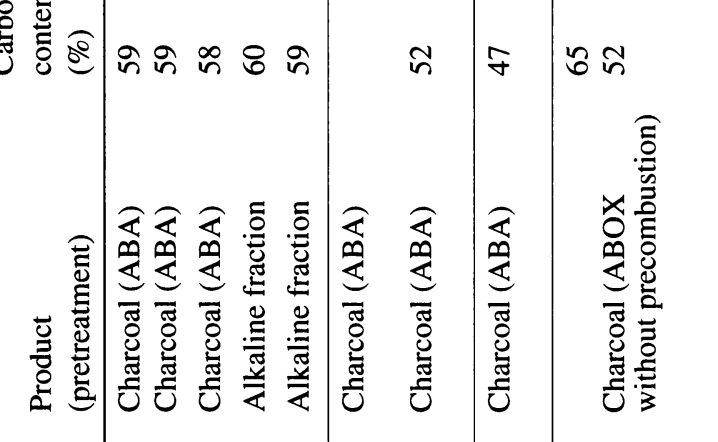

வேं

ల్

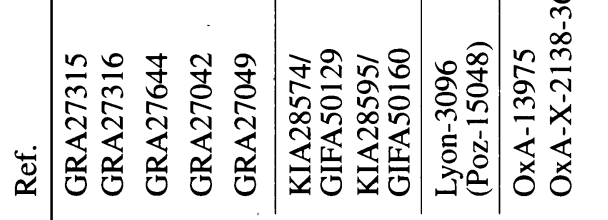

离 


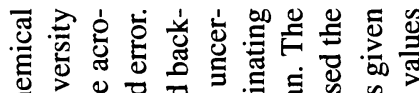

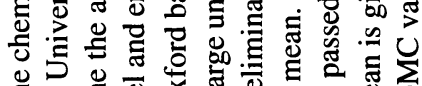
o

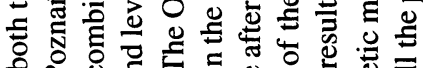
잉

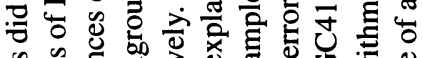

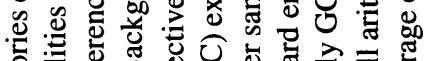

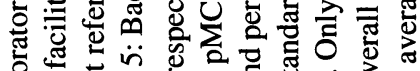

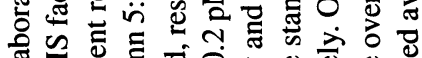

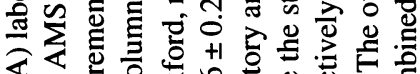
বेष 论 골

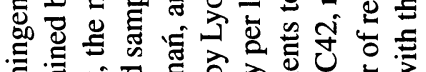

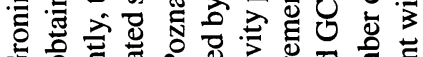

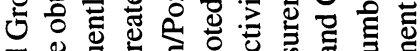
承券

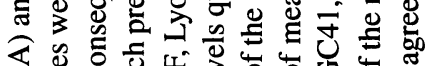
爻记

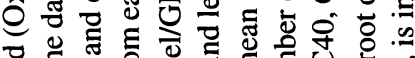
흐을

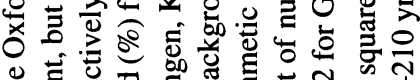

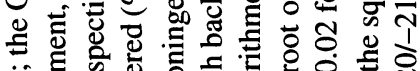
莳镸宫

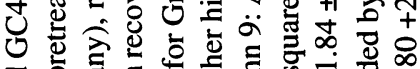

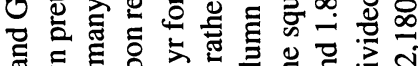
. 结过

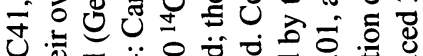

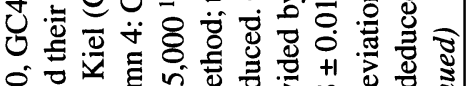
守:

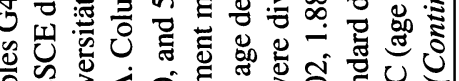

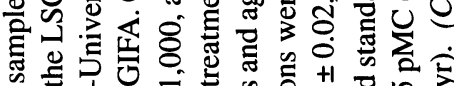

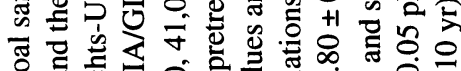

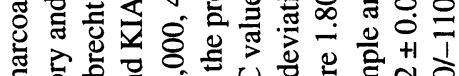

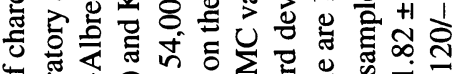

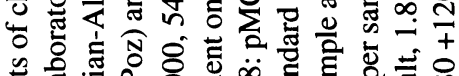

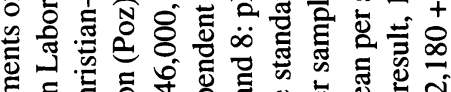

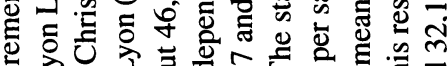

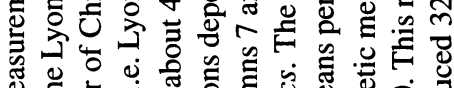

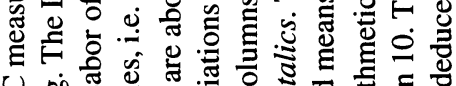
U⿻

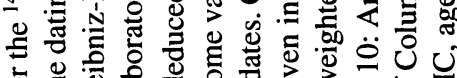

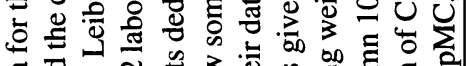

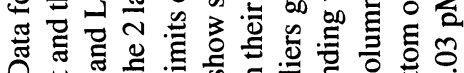

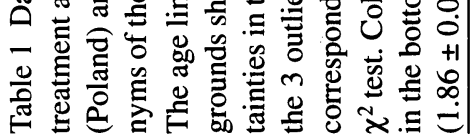

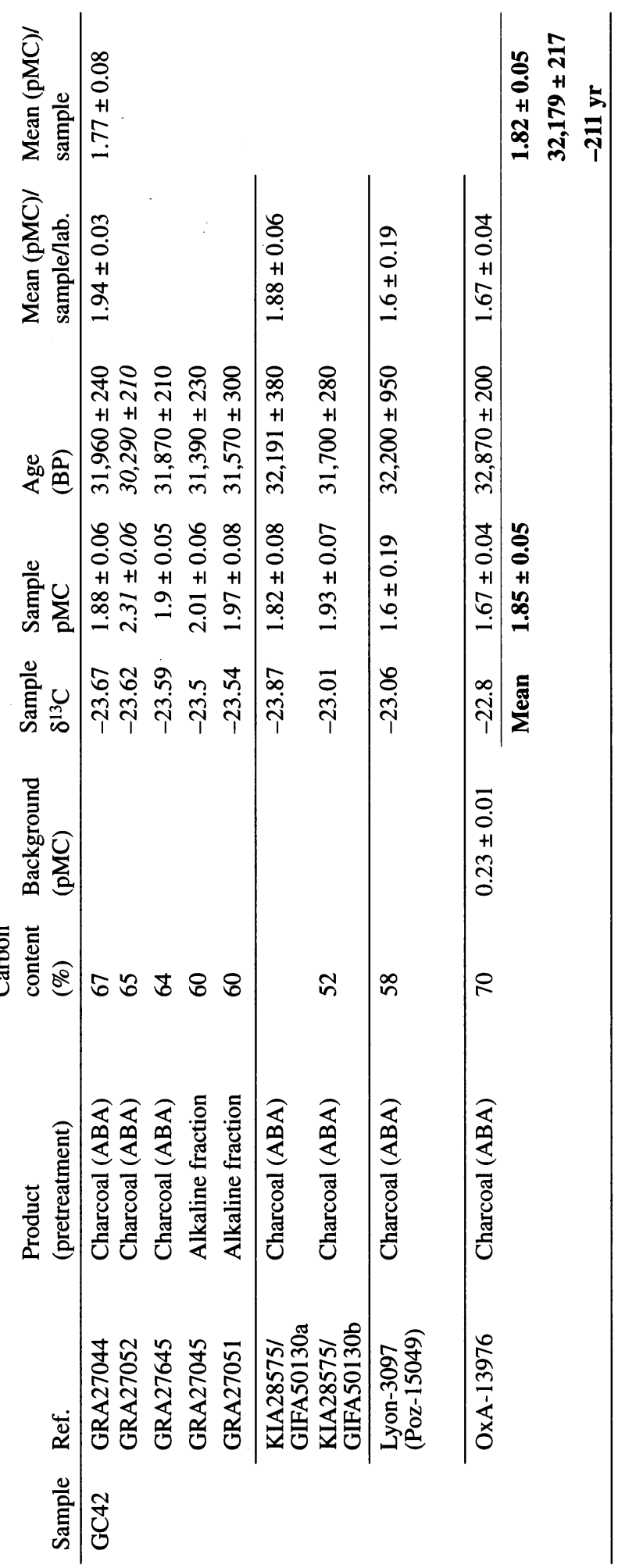


The pMC values for the 3 samples range from 1.7 to just below 2.1 , excluding 1 significantly higher value for each sample $(2.53,3.05$, and 2.31 for GC40, GC41, and GC42, respectively). One can also note that the activities of the alkaline fractions (1.94 and 1.79 pMC for GC41 and 2.01 and 1.97 pMC for GC42; see Table 1, column 7) are in the same range as those measured on the purified charcoal residues of all samples. This agreement between both fractions suggests that GC41 and GC42, as well as GC40, were not contaminated by modern carbon (Batten et al. 1986), and that the ages obtained on the purified charcoal residues are also in this respect reliable. The absence of contamination by extraneous carbon is also apparent from the agreement between the results derived from different pretreatments (with or without precombustion). Therefore, one can infer that the highest pMC value obtained on charcoal residues of GC41 and 42 may be due to some accidental contamination of these samples during the laboratory process; the same could be assumed for the highest pMC value measured on GC40. Consequently, we decided to exclude these 3 outliers from the calculation of the average ${ }^{14} \mathrm{C}$ concentration for each sample when the results of all laboratories are combined. These arithmetic means (and standard errors) are $1.81 \pm 0.05 \mathrm{pMC}, 1.91 \pm 0.03 \mathrm{pMC}$, and $1.85 \pm 0.05 \mathrm{pMC}$ for GC40, GC41, and GC42, respectively; these values are statistically compatible and confirm the assumption that the 3 charcoals collected in the same hearth came from contemporaneous tree specimens. The mean activity deduced for these 3 samples is $1.86 \pm 0.03$ pMC, which yields an age of $32,030 \pm 120 \mathrm{BP}$. A similar result (mean activity: $1.85 \pm 0.03 \mathrm{pMC}$ ) was obtained by averaging all the pMC values, except for the outliers. The fact that the results obtained by the different laboratories for GC40 and GC42 failed the $\chi^{2}$ test can be explained by the fact that the statistical error on the individual results does not represent the total error associated with the date; possible difficulties during sample processing may not have been taken into account in the error calculations.

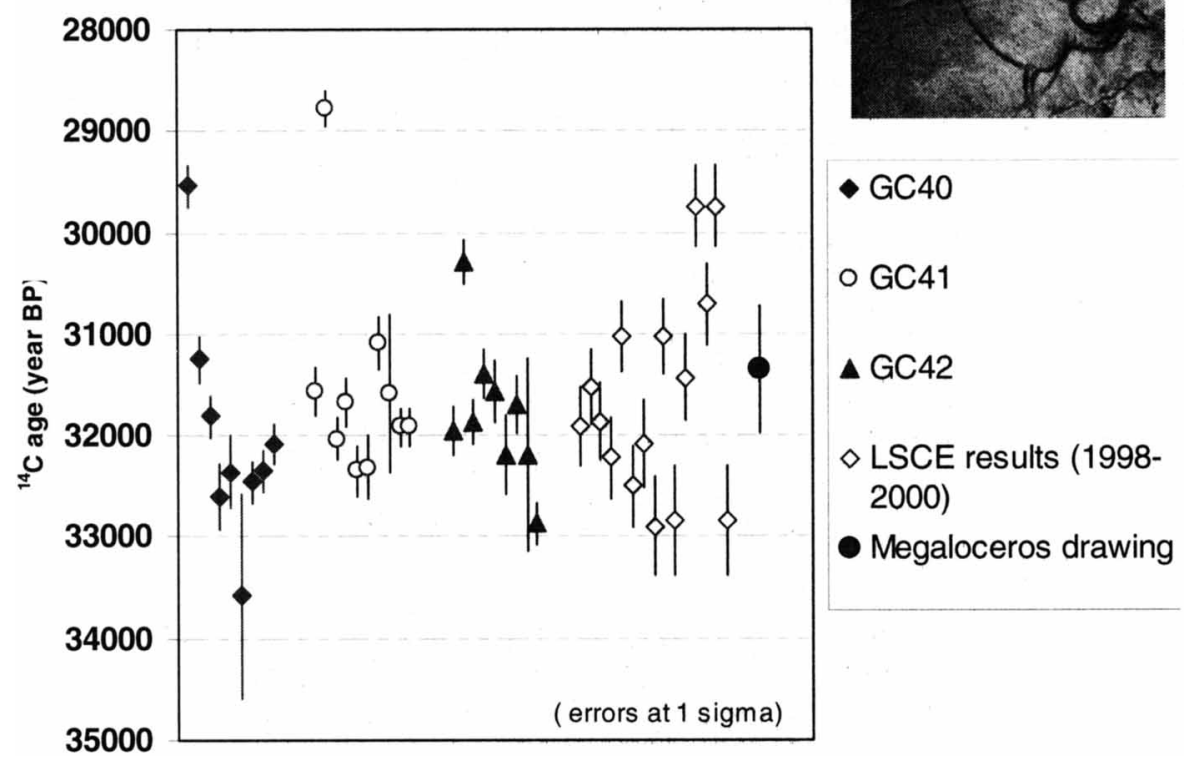

Figure $3{ }^{14} \mathrm{C}$ ages for the charcoal samples. The results obtained for GC40, GC41, and GC42 are indicated by black diamonds, empty circles, and black triangles, respectively. The 15 dates (empty diamonds) previously obtained at the LSCE for several other hearths of the Megaceros gallery are given on the right alongside the age (large black dot) obtained on the megaloceros drawing (picture at top, courtesy of Ministère de la Culture, France). 
One must emphasize that these results cannot be considered as definitive due to the fact that some laboratories have not finished their intercomparison measurements. However, one can already point out that this new composite date for this hearth of the Megaceros gallery is in agreement with the dates previously obtained at the Laboratoire des Sciences du Climat et de l'Environnement (LSCE) on charcoal specimens collected in 10 other hearth structures of the same gallery: $31,600 \pm 1000 \mathrm{BP}$ (arithmetic mean of 15 dates and standard deviation indicating the possible duration of human presence in the Megaceros gallery). This result is also compatible with the ${ }^{14} \mathrm{C}$ age of $31,350 \pm 600 \mathrm{BP}$ obtained on the charcoal used to draw the megaloceros situated at the entrance of the gallery (Valladas et al. 2001, 2005). Finally, the intercomparison program confirms that Chauvet Cave was frequently visited by humans during the Aurignacian period. The presence of cave bears in the cave is also evident during the same period (Bocherens et al. 2006). The relation between the 2 cave visitors remains to be investigated.

\section{CONCLUSIONS}

Charcoal samples from a hearth of the Megaceros gallery in Chauvet Cave were collected and ${ }^{14} \mathrm{C}$ dated as part of an AMS ${ }^{14} \mathrm{C}$ intercomparison program including different pretreatments and fractions from several ${ }^{14} \mathrm{C}$ laboratories. This study clearly demonstrates that several major laboratories can obtain very similar dates for charcoal produced by prehistoric humans more than $30,000 \mathrm{yr}$ ago and answers questions raised about the validity of dates obtained for prehistoric sites in a single laboratory (Pettitt and Bahn 2003). These new dates are in agreement with earlier dates from several other hearths of the Megaceros gallery and direct dates from the paintings from the same sector of the cave, previously measured at the Laboratoire des Sciences du Climat et de l'Environnement. Taken together, these initial intercomparison results provide strong support for the previously published AMS dates from the same parts of Chauvet Cave. The structure of the hearths and the quality and size of the charcoal fragments at the end section of the Megaceros gallery, where hearth \#8 is located, as well as the absence of other remains, suggest that these structures were used for the production of charcoal and not only for domestic purposes (Clottes 2000; Geneste 2001). Unless one assumes that later visitors used ancient charcoal to produce the cave art, the ages obtained here provide the probable age for the execution of the Chauvet cave paintings around 32,000 BP.

\section{ACKNOWLEDGMENT}

The LSCE team wishes to thank Ingrid Caffy for her help with sample preparation.

\section{REFERENCES}

Batten RJ, Gillespie R, Gowlett JAJ, Hedges REM. 1986. The AMS dating of separate fractions in archaeology. Radiocarbon 28(2A):698-701.

Bird MI, Ayliffe LK, Fifield LK, Turney CSM, Cresswell RG, Barrows TT, David B. 1999. Radiocarbon dating of "old" charcoal using a wet oxidation stepped-combustion procedure. Radiocarbon 41(2):127-40.

Bocherens H, Drucker DG, Billiou D, Geneste J-M, van der Plicht J. 2006. Bears and humans in Chauvet Cave (Vallon-Pont-d'Arc, Ardèche, France): insights from stable isotopes and radiocarbon dating of bone collagen. Journal of Human Evolution 50(3):370-6.

Bronk Ramsey C, Higham T, Bowles A, Hedges R. 2004. Improvements to the pretreatment of bone at Oxford. Radiocarbon 46(1):155-63.
Clottes J. 2000. Une journée dans la grotte Chauvet. In: Grandes girafes et fourmis vertes. Paris: La Maison des Roches. p 181-2. In French.

Clottes J, editor. 2001. La Grotte Chauvet. L'art des origines. Paris: Seuil. 224 p.

Clottes J, Chauvet J-M, Brunel-Deschamps E, Hillaire Ch, Daugas J-P, Arnold M, Cachier H, Évin J, Fortin $\mathrm{Ph}$, Oberlin Ch, Tisnérat N, Valladas H. 1995. Les peintures paléolithiques de la grotte Chauvet-Pont d'Arc (Ardèche, France): datations directes et indirectes par la méthode du radiocarbone. Comptes rendus de l'Académie des Sciences de Paris 320 (series IIa): 1133-40. In French.

Cohen-Ofri I, Weiner L, Boaretto E, Mintz G, Weiner S. 2006. Modern and fossil charcoal: aspect of structure 
and diagenesis. Journal of Archaeological Science 33(3):428-39.

Geneste J-M. 2001. Les fréquentations et les activités humaines. In: Clottes J, editor. La Grotte Chauvet. L'art des origines. Paris: Seuil. p 44-7. In French.

Geneste J-M, director. 2003. Recherches pluridisciplinaires dans la grotte Chauvet. Journées SPF, Lyon, 1112 October 2003. Société Préhistorique Française, Travaux 6, T. 102, nr 1. In French.

Pettitt PB, Bahn P. 2003. Current problems in dating Palaeolithic cave art: Candamo and Chauvet. Antiquity 77(295):134-41.
Valladas H, Clottes J, Geneste J-M, Garcia MA, Arnold M, Cachier H, Tisnérat-Laborde N. 2001. Palaeolithic paintings: evolution of prehistoric cave art. Nature 413(6855):479.

Valladas H, Tisnérat-Laborde N, Cachier H, Kaltnecker E, Arnold M, Oberlin C, Evin J. 2005. Bilan des datations carbone 14 effectuées sur des charbons de bois de la grotte Chauvet. Bulletin de la Société Française de Préhistoire 102(1):109-13. In French.

Züchner Ch. 1996. The Chauvet Cave: radiocarbon versus archaeology. International Newsletter on Rock Art 13:25-7. 